\title{
Colistin's dark face. Resurrection of polymyxin antibiotics and alternative strategies
}

\begin{abstract}
Due to the lack of new antibiotics for the treatment of critically ill patients with multidrugresistant bacteria, interest on "old" antibiotics like colistin (polymyxin E) re-emerged. This polymyxin has gained an important role as salvage therapy for patients infected with microorganisms susceptible only to colistin. It is important to know the dark face of colistin referring here to its important toxicity, often lethal, especially in critically ill patients. Given its rarity we briefly mentioned a case of profound encephalopathy in a post-surgical patient who recovered completely after stopping the drug. In addition, its variable pharmacokinetics make it very difficult to establish an effective regimen, most of which is infratherapeutic and with the limitation of its toxicity, which is a terrible problem for humanity. The common sense of the clinician and the birth of new antibiotics, as an alternative strategy, represent a hope to eradicate multiresistance.
\end{abstract}

Keywords: colistimethate Sodium, multidrug-resistant bacteria (MDR), encephalopathy, extended spectrum beta lactamase (ESBL); B-lactamase inhibitors
Volume 8 Issue 6 - 2017

\section{Fernández Ana Belén}

Staff of Anesthesia, Department of Anesthesiology and Intensive Care, Ntra Sra De Candelaria University Hospital, Spain

Correspondence: Fernández Ana Belén, Staff of Anesthesia, Department of Anesthesiology and Intensive Care, Ntra Sra De Candelaria University Hospital, Spain, Email anabfb@gmail.com

Received: April 29, 2017| Published: October 12, 2017

\section{Introduction}

Due to the lack of new antibiotics for the treatment of critically ill patients with multidrug-resistant bacteria, interest on "old" antibiotics like Colistin (polymyxin E) re-emerged. Colistin was initially used therapeutically in Japan and in Europe during the 1950s and in United States in the form of Colistimethate Sodium (CMS) in 1959. However, the intravenous formulations of colistin and polymyxin B were gradually abandoned in most parts of the world in the early 1980s because of the reported high incidence of adverse effects, nephro- and neurotoxicity. Colistin, is currently used as a last-line drug for the treatment of MDR Gram negative bacteria infection, so in the last fifteen years, this polymyxin has gained an important role as salvage therapy for patients infected with microorganisms susceptible only to colistin. ${ }^{1-4}$ There is a dearth of information on the pharmacodynamics and toxicodynamics of colistin and its non-active prodrug CMS. Optimised dose regimens have not been establishment for different types of patients, especially in the critically ill population.

\section{Material and methods}

Regarding the metaphorical use of the dark face of colistin, we talk about its nephro and mainly neurotoxicity, especially in our post-surgical patients, moderate and severe encephalopathy and life-threatening respiratory paralysis caused by neuromuscular blockage. Recents reports have not described neurotoxicity associated with intravenous CMS, with the exception of cases in the cystic fibrosis population, among whom neurotoxicity has manifested as paresthesias and ataxia in $29 \%$ of patients treated (dosages in excess of $5 \mathrm{mg} / \mathrm{kg}$ per day). Our patient received intravenous CMS dosages $6 \mathrm{mg} / \mathrm{kg}$ per day. Only one case according most recent literature have described a profound encephalopathy with lack of brainstem reflexes in postsurgical patient. ${ }^{5}$ 56-year-old man who developed renal, hematological and neurotoxicity on the 5 day after administration endovenous colistin and cefepima (CMS dosages of $160 \mathrm{mg} / 8 \mathrm{~h}$, equivalent to approximately $6 \mathrm{mg} / \mathrm{Kg}$ per day). The patient has made perforated diverticulitis with descending colon resection and end colostomy left flank. On day 6 post-surgery nosocomial pneumonia by Pseudomona Aeruginosa MR sensible to colistin was diagnosed.
Electroencephalogram was compatible with severe encephalopathy. Peripheral eosinophil count had increased to $12 \%$. He recovered promptly after stopping the drug.

\section{Results and discussion}

The awareness of colistin's potentially fatal effects must be kept in mind when we using. Vigilance of the encephalopathic picture can also facilitate the diagnosis of colistin-mediated neurotoxicity in a patient with altered mental status of otherwise unknown aetiology. The establishment of any relationships between the daily dosage of CMS, colistin levels, and neurological events is needed, especially in the critically post-surgical ill patient population.

It is contradictory that from the study of the most current works on colistin we can deduce that we are not using the correct dose: relatively poor potency and lung penetration, variable PK. ${ }^{6}$ Dosing regimens do not allow high probability of achieving optimal exposures and we can't increase dose due to toxicity so we get low PD exposures. Are postulated as probably more effective dose: loading dose of 9 MIU followed by a maintenance dose of $4.5 \mathrm{MIU} / 8-12 \mathrm{~h}{ }^{7}$

Finally, the increased use of colistin has resulted in the development of colistin-resistance. Adaptative or mutational mechanisms may play a role in resistance to colistin. Different gene mutations cause resistance by changing the outer membrane of gram-negative bacteria. Colistin resistance in Pseudomona Aeruginosa has been reported as 3\% in the literature, no case in critical post-surgical patients. ${ }^{8-10}$ Multidrug resistant $P$ aeruginosa often requires complex antimicrobial regimens and, when treated inadequately, infections caused by this pathogen are associated with particularly poor outcomes including postoperative complications, longer hospital stays, and increased mortality. Until recently, ceftazidime was considered the most active cephalosporin against $\mathrm{P}$ aeruginosa strains; however this antimicrobial agent is affected by upregulation of efflux systems and by the acquisition of beta-lactamases.

Ceftolozane-tazobactam is an antibacterial consisting of ceftalozane, an antipseudomonal cephalosporin that has improved stability against the chromosomal AmpC produced by P Aeruginosa, 
and tazobactam, a penicillanic acid-sulfone beta-lactamasa inhibitor. Ceftolozane-tazobactam has been shown to be more active against resistant gram negative bacteria, especially P. aeruginosa compared to cephalosporins, carbapenems and piperacillin-tazobactam, also in ESBL-producing enterobacteriaceae. On the other hand, Avibactam is a new inhibitor of beta-lactamases. In vitro, it inhibits Ambler A class $\mathrm{C}(\mathrm{Amp} \mathrm{C})$ enterobacteria and some class D enzymes (including OXA 48 , except those produced by A Baumannii). Avibactam improves the activity of ceftazidime against ESBL-producing gram negative bacteria, carbapenemase type A (KPC), some type D (OXA) and class $\mathrm{C}$ of Ambler (AmpC). ${ }^{11-13}$

\section{Conclusion}

At the present time, the use of any antibiotic is associated with the potential development of tolerance or resistance from the first moment it is used. The burden of antibiotic resistance in gram-negative bacteria increases exponentially throughout the world and represents a global health problem. It is crucial at this time to optimize antibiotics and their rational use. With the birth of the new beta-lactam/beta-lactamase inhibitors, Ceftalozane-tazobactam and Ceftazidime-avibactam, we can talk about a new horizon to combat multi-resistance in our time.

\section{Acknowledgments}

None.

\section{Conflicts of interest}

Author declares that there is no conflict of interest.

\section{References}

1. Bosso JA, Liptak CA, Seilheimer DK, et al. Toxicity of colistin in cystic fibrosis patients. DICP. 1991;25(11):1168-1170.

2. Fernández AB, Perez M, Soto L. Sudden respiratory muscle paralysis and apnea in a patient infected with multidrug-resistant Pseudomonas Aeruginosa treated iv colistin. IJID. 2013;17:e357.
3. Myint T, Evans ME, Burgess DR, et al. Respiratory Muscle Paralysis Associated With Colistin, Polymyxin B, and Muscle Relaxants Drugs: A Case Report. J Investig Med High Impact Case Rep. 2016;4(1):2324709616638362.

4. Nigam A, Kumari A, Jain R, et al. Colistin neurotoxicity: revisited. $B M J$ Case reports; 2015.

5. Wadia S, Tran B. Colistin-mediated neurotoxicity. BMJ case Reports; 2014.

6. Trifi A, Abdellatif S, Daly F, et al. Efficacy and Toxicity of HighDose Colistin in Multidrug-Resistant GramNegative Bacilli Infections: A Comparative Study of a Matched Series. Chemotherapy. 2016;61(4):190-196.

7. Nation RL, Garonzik SM, Li J, et al. Updated US and European Dose Recommendations for Intravenous Colistin: How Do They Perform? Clin Infect Dis. 2016;62(5):552-558.

8. Matthaiou DK, Michalopoulos A, Rafailidis PI, et al. Risk factors associated with isolation of colistin-resistant gram-negative bacteria: a matched case-control study. Crit Care Med. 2008;36(3):807-811.

9. Pitt TL, Sparrow M, Warmer M, et al. Survey of resistance of Pseudomona Aeruginosa from UK patients with cystc fibrosis to six commonly prescribed antimicrobial agents. Thorax. 2003;58(9):794-796.

10. Yilmaz GR, Dizbay M, Guven T, et al. Risk factors for infection with colistin-resistant gram-negative microorganisms: a multicenter study. Ann Saudi Med. 2016;36(3):216-222.

11. Solomkin J, Hershberger E, Miller B, et al. Ceftolozane/Tazobactam plus Metronidazol for Complicated Intra-abdominal Infections in an Era of Multidrug-Resistance: Results From a Randomized, Double-Blind, Phase 3 Trial (ASPECTcIAI). Clin Infect Dis. 2015;60(10):1462-1471.

12. Salgado P, Gilsanz F, Maseda E. Resistant gram-negative bacteria. Therapeutic approach and risk factors. Rev Esp Quimioter. 2016;29(Suppl 1):26-30.

13. Tato M, Garcia-Castillo M, Moreno A, et al. In vitro activity of ceftolozane/ tazobactam against clinical isolates of Pseudomonas Aeruginosa and Enterobacteriaceae recovered in Spanish medical centres: Results of the CENIT study. Int J Antimicrob Agents. 2015;46(5):502-510. 